\section{Military Technical College \\ Kobry El-Kobbah, Cairo, Egypt.}

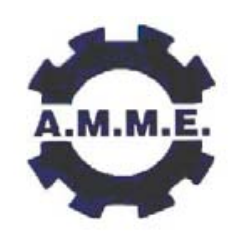

\title{
IMPACT DAMAGE PREDICTION IN SHELL STRUCTURES
}

\author{
EI-FARRAN* M.M., ABUELFOUTOUH** N.M. and NEGM** H. M.
}

\begin{abstract}
This paper describes a method for predicting how much impact kinetic energy is needed to deform, penetrate, and perforate a shell structures. This method based on strain rate effects, non linear materials, and cross section area reduction. An explicit finite element solver was developed to investigate the relationship between the impactor and target under different impact load cases. The study shows that the impactor can deform, penetrate, and perforate the structure depending on the shape, and the velocity of the impactor besides the material properties of both the impactor and the structure. This paper considers a shell structure response subjected to rigid spherical impactor with a fixed weight at different impact speeds ranging between 10 $\mathrm{m} / \mathrm{s}$ to $150 \mathrm{~m} / \mathrm{s}$.
\end{abstract}

\section{KEY WORDS}

Impact, structure, damage, shell, sphere, contact, strain rate, Non-Linear Material

\footnotetext{
Ph.D. Student in Aircraft Structure, Department Of Aerospace Engineering, Cairo University

** Professor of Aircraft Structures, Department Of Aerospace Engineering, Cairo University

*** Professor of Aircraft Structures, Department Of Aerospace Engineering, Cairo University
} 


\section{INTRODUCTION}

The impact of deformable bodies is a much complicated event than Rigid and elastic impact. The short duration and large localized of the stresses generated in the vicinity of the contact area produces plastic deformation in most practical impact problems. Rigid and elastic impact are engineering applications that are acceptable only at relatively low impact speeds.

Of general interest in engineering is the coefficient of restitution from which the kinetic energy loss during an impact is estimated. The dissipation of kinetic energy associated with impact can be attributed mainly to two causes. First, the propagation of stress waves produced by the impact and second, the plastic deformation developed in the contacting bodies during the impact. The energy losses due to elastic wave propagation during an elastic impact were theoretically investigated by Hunter [2] who showed that for a steel ball impinging on a large block of steel or glass, less than 1 per cent of the kinetic energy of the ball is converted into elastic waves. A re-evaluation by Reed [9] predicted the energy loss as being some 4.5 times greater than that estimated by Hunter. Tillett [10] experimentally investigated the energy loss due to stress wave propagation and found that the energy losses were of the order of 3 per cent for a steel ball impacting on glass.

Hutchings [3] theoretically analyzed the energy dissipation by elastic waves during plastic impact and showed that for the impact of hard steel spheres on mild steel targets with velocities, around $70 \mathrm{~m} / \mathrm{s}$, the energy lost by elastic wave propagation is also about $3 \%$ of the initial kinetic energy. Thus only a very small portion of the kinetic energy is generally dissipated by stress wave propagation.

At high impact velocities, the dominant energy dissipation mechanism is plastic deformation. The energy losses due to plastic deformation have recently received considerable attention in both theoretical and experimental studies. Several models have been proposed to predict the coefficient of restitution for the plastic impact of a sphere with a surface [4]. However, due to the complicated mechanism of plastic impact, all of these models are based on some simplifying assumptions and need to be tested extensively by experimental and numerical data. Some experimental results have been reported in [5] but the range and reliability of these results are limited. Although a considerable number of studies have been conducted to investigate the impact phenomena of elastic-plastic spheres, understanding of the rebound characteristics is far from complete.

The aim of the present work is to investigate the effect of impactor velocity on the plastic target deformation using finite element methods. Focusing on the prediction of the needed kinetic energy to reach which level of deformation, various impacts of a sphere against a shell have been modeled, in which different impact velocities in the range from the yield velocity to the limit of shell perforation are applied. The finitedeformation plastic impact is addressed and the contact effect is presented. The yield velocity is the impactor velocity which makes the target deformation be a plastic deformation. 


\section{FINITE-ELEMENT MODELING}

The main problem here is a flat plate "target" impacted by a rigid spherical impactor. The proposed finite-element grid is shown in Fig.1. The target is a steel sheet of dimensions of $150 \mathrm{~mm} \mathrm{X} 150 \mathrm{~mm}$ and $2.5 \mathrm{~mm}$ thickness and is modeled using Belytschko-Lin-Tsay [7] shell elements. It is clamped at all edges and subjected to impact by a rigid spherical steel projectile of $20 \mathrm{~mm}$ radius, $2.5 \mathrm{~mm}$ thickness and 152.1 $\mathrm{gm}$ mass at a speed range from 10 to $150 \mathrm{~m} / \mathrm{s}$.

The projectile is modeled as Belytschko-Lin-Tsay shell elements using rigid body element, which is suitable for large displacement modeling. Both the target and sphere are modeled in full so as to be able to simulate the stress waves propagating in the target from the point of impact towards the clamped edges and back. This will affect how the target will respond to impactor. In the analysis, the length and width of the specimen are considerably larger than its thickness $(2.5 \mathrm{~mm})$.

\section{Material Model}

The material used here for the target plate is a steel elasto-plastic material. This material has a stress-strain relationship presented in Fig.2. The material includes a strain rate effect, which will be described in the next section. The material stress response depends on the rate of strain application. This model applied provides linear unloading for the material.
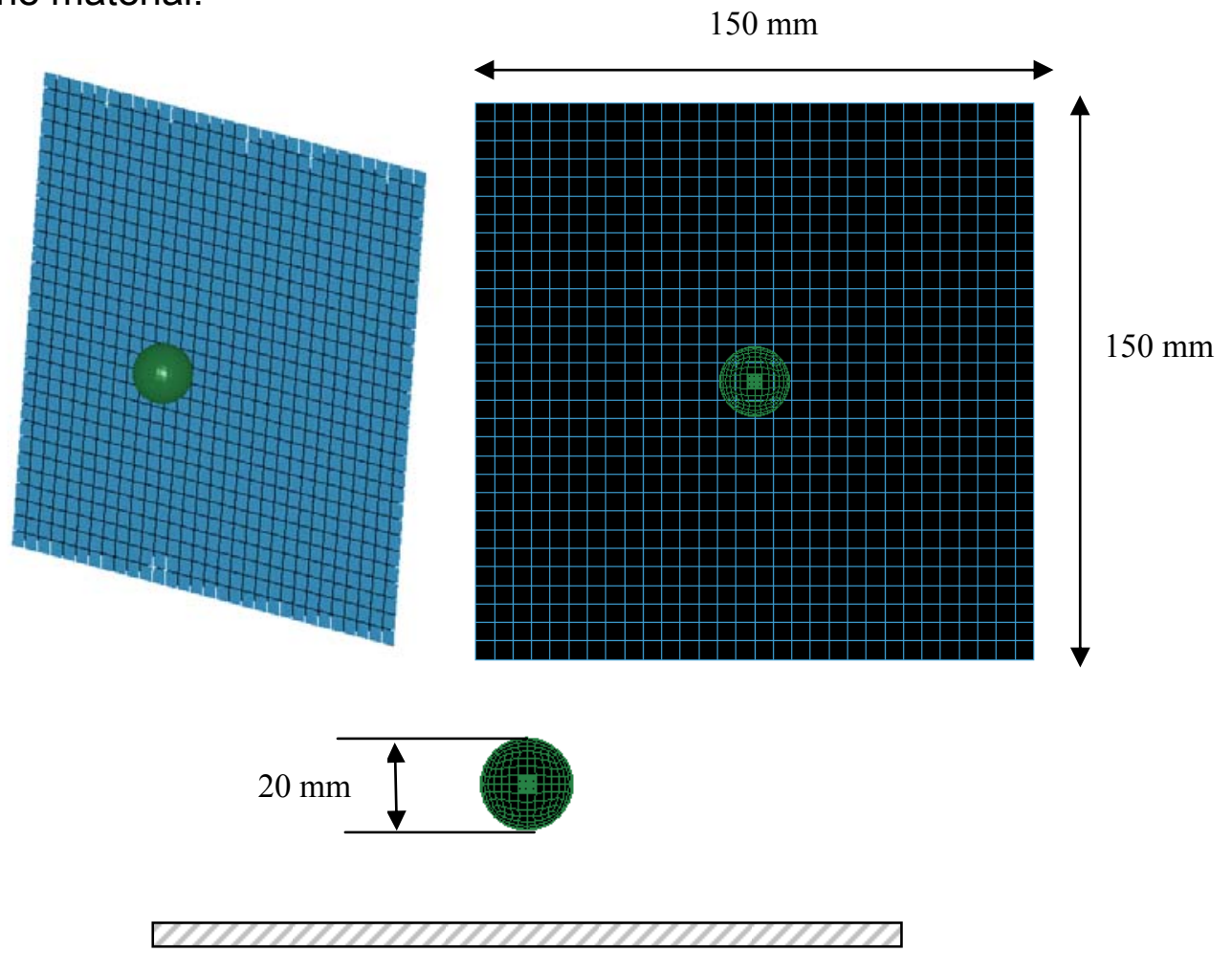

Fig.1. Finite-element model of the impactor and target. 


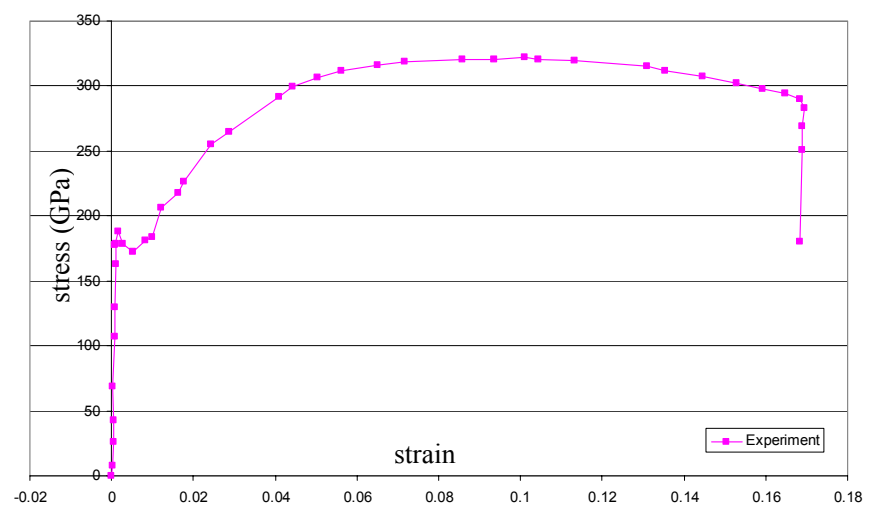

Fig.2. The stress-strain relationship of the steel

The impactor material is assumed rigid and is represented in the finite element model by the Belytschko-Lin-Tsay rigid body shell element

\section{Stress-Strain Rate Dependence}

Impact of structures is a high strain loading of the impacted structures. Most materials at high strain rates show stress-strain rate dependence. The stress-strain rate dependence of the impacted structure implies that the material stress response changes with the applied strain rate. To account for this effect in the structural nonlinear stress calculation, a method is developed for scaling the forces based on the relative velocities that apply to all active contacted elements. The forces are computed from element deformation by applying the elements material properties. These forces are then scaled by an amplification factor to obtain the dynamic value. The shell structure material has a non linear behavior. This would allow the prediction of the shell perforation ad penetration under impact load [7]. Where the equation describe this relation is

$$
F_{\text {dynamic }}=\left(1+K_{d} \frac{V}{V O}\right) F_{\text {static }}
$$

where

$k_{d} \quad$ is a user defined input value

$V \quad$ is the time relative velocity

$V_{0} \quad$ is the dynamic test velocity

And in this paper, $\mathrm{Kd}$ equals $1 \mathrm{e}-6$ is used.

\section{Contact Detection}

The dynamic stress state at the time period of contact between the impactor and target is solved as a contact problem. Finite element methods have been used for many years to solve contact problems. Contact problems are inherently non-linear since, prior to contact, boundary conditions are given by traction boundary conditions, in which the traction normal to the target surface is often zero before impact. During contact, kinematical constraints are imposed which prevent penetration of one boundary into the other, which is the impenetrability condition. The solution of a contact problem involves 
first identifying which points on the boundaries interact with each other, and seconds the insertion of appropriate conditions to prevent penetration [4].

There are two major classes of contact finite element algorithms. One searches the nodes and checks them against a surface to determine if contact is imminent. The second class searches element surfaces and checks them against other surfaces (and edges) to determine the shortest distance and thereby if contact is imminent. The element-to-element concept is a bit trickier to implement, and depends on the elements shape and type. The node-to-element type is simpler in programming and well documented, and it will be the one to be used in the present analysis.

The distance from a node to a surface can be tricky to calculate if the surface is complex. If, however, the surface is a simple triangle, it is just a matter of linear algebra. A general method which works for different types of elements is wanted. These two reasons have resulted in a concept where contact "finite surfaces" is used. Any element uses one or several contact surfaces to handle the contact with other elements (or rather nodes). When an element is investigated to check its contact, the element in turn investigates each of its surfaces to check for contact. The resulting contact forces are then distributed onto the element nodes [7].

As an application, the Belytschko-Lin-Tsay Quad shell element already used above will be considered in the impact analysis. Since this element is flexible and can bent, two flat triangular contact surfaces cannot accurately model the shell element. In this case, as shown in Fig.3, four contact triangles would be suitable. Each one of them is connected to a centre point on the shell element and two corner nodes. This procedure could represent the curvature in a rough but simple way, and even allow for self contact, i.e. when the element wraps around and contacts itself. This allows for the prediction of structural crash where the structural shell elements may fold around and contact itself.

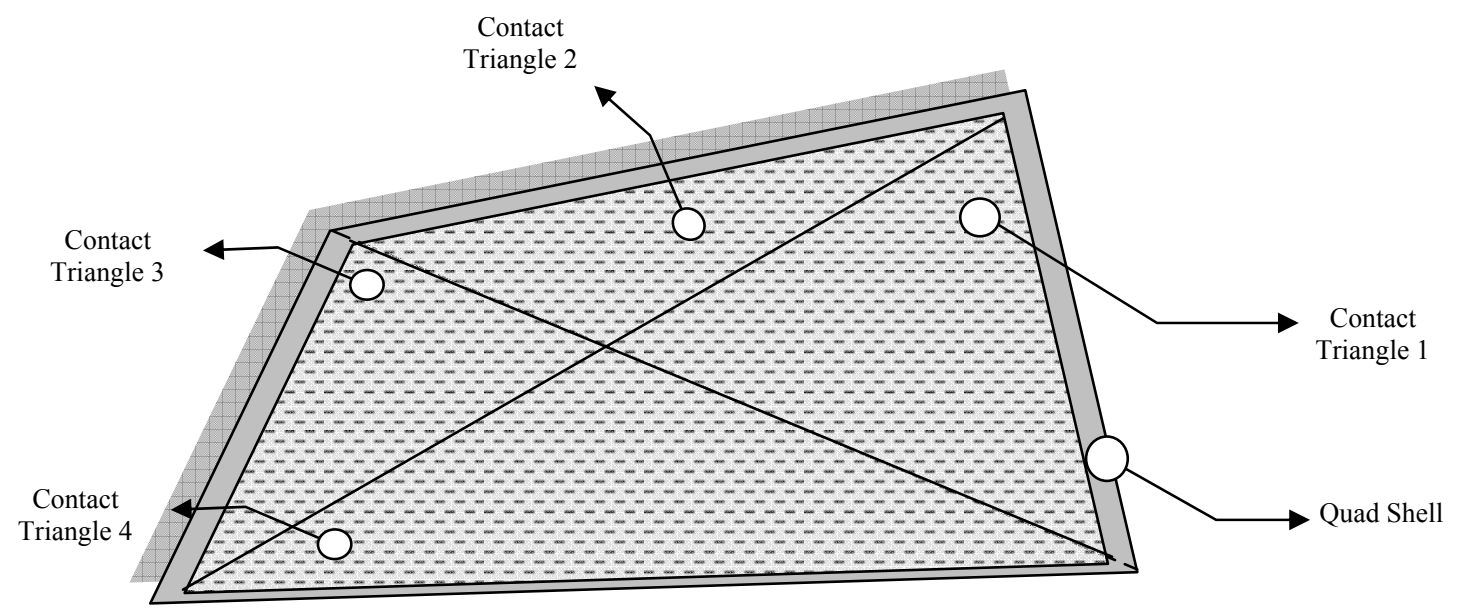

Fig.3. BLT Quad shell and contact triangles

\section{Impactor Force Calculation}

Three distinct methods for handling sliding and impact along impacted surfaces are investigated in this research. First, the kinematics constraint method, second, the 
penalty method and third, is the distributed parameter method [5]. The third method was not considered in the since it is useful only in the sliding and friction calculation, and not mainly for impact. To investigate the relative merits of the other two approaches the impacted Interfaces are defined in three dimensions by listing in an arbitrary order all triangular and quadrilateral segments that comprise each side of the interface. One side of the interface is designated as the slave side, and the other as the master side. Nodes lying in those surfaces are referred to as slave and master nodes, respectively.

In the symmetric penalty method, this distinction is was found irrelevant. In the kinematics constraint method the slave nodes are constrained to slide on the master surface after impact and remain on the master surface until a tensile force develops between the node and the surface. This tensile force indicated the separation on the impacted surfaces.

The contact force is calculated from the deformation of the master and slave nodes by applying the following equation [7]:

$$
F_{r}=\left(C_{R F}\right) * E *\left(1-\frac{D}{R}\right)
$$

Where

$$
\begin{aligned}
& \mathrm{C}_{\mathrm{RF}} \quad \text { : } \quad \text { Contact Reaction Factor, which is related to the materials of } \\
& \text { the collided objects. } \\
& \text { E : Slave object Material Young's modules } \\
& \text { D : : Distance between the collided nodes } \\
& \mathrm{R} \quad \text { : } \quad \text { Contact sphere radius }
\end{aligned}
$$

And Frictional Force [7]:

$$
F_{r}=\left(C_{F F}\right) * E *\left(1-\frac{D}{R}\right)
$$

where

$\mathrm{C}_{\mathrm{FF}}: \quad$ Fraction Factor, which is related to the materials of the collided objects.

Automatic definitions of the slave and master surfaces are generated internally by the routine ID for each surface [7]. For crash models it is quite common to include the entire model in one single surface contact definition where all the nodes and elements within the interface can interact.

\section{Effect of Time Step Scale Factor on Numerical Solution Stability}

For numerical stability, the step size $\Delta t$ for each time increment in the simulation should be smaller than the time for a stress wave to move across the smallest finite element in the mesh. For impact problems that involve large deformation, this limiting time step $\Delta t$ varies throughout the simulation. In two dimensions, this relationship is governed by

$$
d=\frac{1}{k} c \Delta t
$$


where $\mathrm{d}$ is the distance across the smallest finite element, $\mathrm{c}$ is the tensile wave speed of the material ( $\sqrt{E / \rho}$ ); where, $\mathrm{E}$ is Young's modulus, $\rho$ is structure material density and $\mathrm{k}$ is the time step scale factor (TSSF). Here, it has a default of 0.9 .

Theoretically, the time step-sizing algorithm in the program would provide an optimum time step just below the limiting value to ensure stability and to minimize the cost of the analysis. The default TSSF of 0.9 enhances numerical stability. However, simulation results obtained using elasto-plastic materials indicate that the stability of the analysis is highly dependent on the TSSF used [3]. This would imply that appropriate selection of the TSSF has to be made for every impact velocity. TSSF of 0.162 has been used by some authors [3] to gives the most accurate residual velocity as compared with that from experiment.

\section{Mesh Intensity}

In elastic structures, the solution of Finite element method converges to a better result as the Finite element mesh is refined. In the rate-dependent nonlinear structure solutions, refining the mesh is not always advantageous as it might lead to numerical instability that provides a divergent solution. It might also lead to numerical error accumulation that might lead to a less accurate solution that that obtained by a less refined mesh. It is also noticed that when using a much course mesh size, the solution again becomes unstable and failure always occurs along the line of highest node density. This might be attributed to the representation of a complex problem by a few pieces of information at little number of nodes. As such the solution can not trace the actual material response. The choice of an optimum mesh for the rate dependent transient nonlinear finite solution depends on the time step. In order that the stress wave recognizes the finite element, the time step multiplied by the speed of stress wave propagation must be less than the mesh size.

In an effort to determine the optimum mesh spacing for the model, numerical results have been obtained for different uniform mesh sizes and compared with each other data. The results obtained suggest that the mesh spacing necessary to simulate a particular impact problem accurately is not arbitrary; also, it does not converge with higher mesh density, but must be chosen depending on the shape, size and mesh density of the projectile. The effects of mesh size on results have also been reported by Billon [1] in his numerical model for ballistic impact.

A good mesh size of 900 quad BLT shell elements $(30 \times 30)$ is used for the target and 384 BLT shell elements are used to model the rigid projectile as it gives the best spherical shape of the target. The following analysis of the results obtained is based on this mesh configuration.

\section{RESULTS AND DISCUSSION}

The results obtained in this research structural rate dependent impact model is compared with those obtained from the numerical solution of the LS-Dyna structural dynamics model in terms of residual velocities, energy absorption and displacement profiles. 


\section{Residual Velocity}

Figure 4 shows good agreement between the two numerical solutions in the calculated residual velocities.

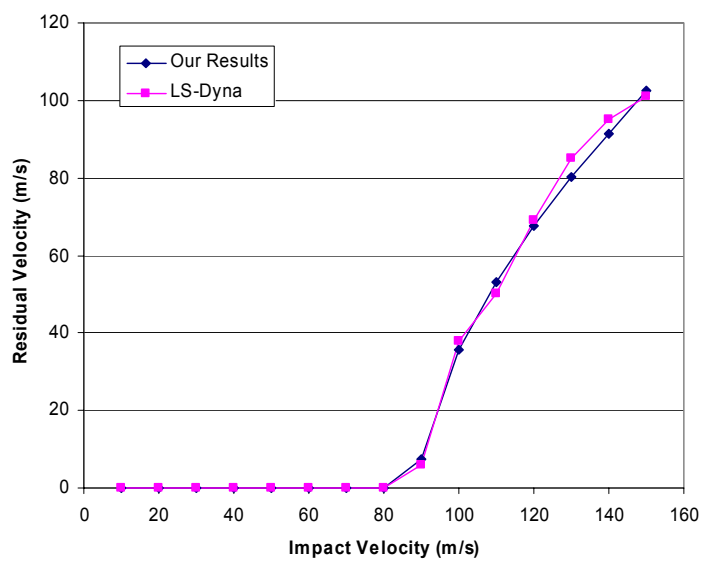

Fig.4. Projectile residual velocity as a function of impact velocity.

\section{Energy Absorption}

The proposed model is able to account for the sharp drop in energy absorbed by the target at high impact velocities. Generally, variation of energy absorbed with impact velocity can be divided into three regions,

Region (1)_Impact velocities lower than ballistic limit: Below the ballistic limit, the projectile did not penetrate the target. Hence, the energy absorbed by the target is equal to the total kinetic energy possessed by the projectile.

Region (2)-Impact between the ballistic limit and critical velocity at which the perforation occurred: This region is considered the "low" velocity impact region, where the initial increase in stress is insufficient to attain the fracture stress of the target. Hence, the transverse deflection has time to propagate to the edges.

The behavior of the target within this region is denoted by extensive creasing and stretching. Energy absorbed increases with impact energy up to a maximum critical velocity.

Region (3)-Impact above the critical velocity: When the impact velocity is above the critical velocity, the target is rapidly penetrated and energy absorbed is very small and becomes approximately constant.

\section{Target Displacement Profile}

When a projectile impinges on a target, stress waves that originate from the impact point move along the material to the edges of the target where they are reflected. Moreover, during the perforation process, the level of strain and rate of strain changes considerably with time. Hence, the material properties which are functions of strain rate vary throughout the entire process. The behavior of target under transverse impact therefore involves a complex relationship of the rate dependent stress-strain properties 


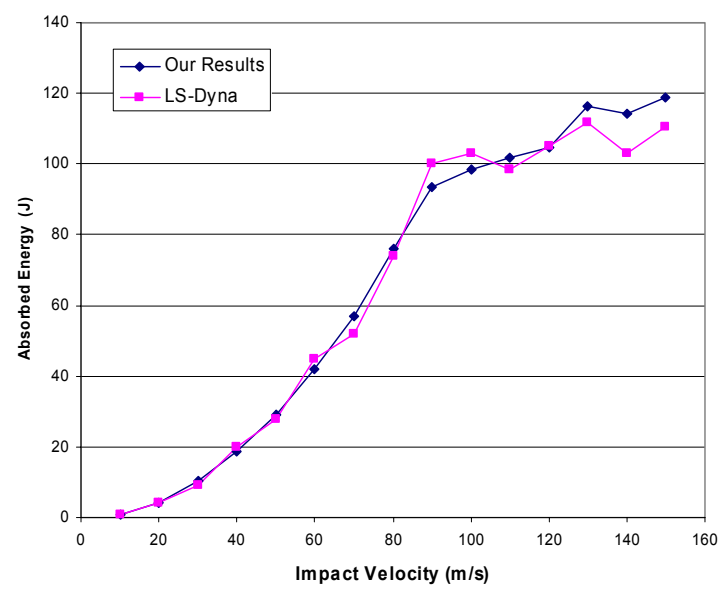

Fig.5. Variation of energy absorbed by the target with impact velocity.

and stress wave interference. The change in deformation of the target with impactor velocity obtained using the proposed model is shown in Fig. 6 for impactor velocities equals $10,20,30,60,90,150 \mathrm{~m} / \mathrm{s}$, respectively.

At speed of $10 \mathrm{~m} / \mathrm{sec}$ the shell deforms elastically and the impactor re-bounces back after $202 \mu \mathrm{Sec}$ with most of its kinetic energy. Only the part of energy that goes into the elastic waves in the shell is subtracted from that of the re-bouncing impactor. The shell returns to its original position due to unloading.

At speed $20 \mathrm{~m} / \mathrm{sec}$, the shell deforms plastically leading to local perforation. The impactor re-bounces back with much less kinetic energy. The part of energy that goes into the elastic waves in the shell and the energy for permanent deformation of the shell are subtracted from that of the re-bouncing impactor. Also, the shell does not return $t$ its original position as it has a permanent deformation.

At speed $30 \mathrm{~m} / \mathrm{sec}$, the some of the shell elements around the point of impact fracture and the shell is penetrated partially by the impactor. The size of the fractured elements did not allow the impactor to pass through. The impactor is captured in the shell and it delivers all its kinetic energy to the fractured elements and to the permanent deformation of the surrounding shell elements. Besides the part of energy that goes to the elastic wave propagation in the shell.

At speed $60 \mathrm{~m} / \mathrm{sec}$, more of the shell elements around the point of impact fracture but the shell is penetrated only partially by the impactor. The size of the fractured elements still did not allow the impactor to pass through. The impactor is captured in the shell and it delivers all its kinetic energy to the fractured elements and to the permanent deformation of the surrounding shell elements. Besides the part of energy that goes to the elastic wave propagation in the shell.

At speed $90 \mathrm{~m} / \mathrm{sec}$, more and more of the shell elements around the point of impact fracture but the shell is penetrated still only partially by the impactor. The size of the fractured elements still did not allow the impactor to pass through. The impactor is captured in the shell and it delivers all its kinetic energy to the more fractured elements and to the permanent deformation of the surrounding shell elements. Besides the part of energy that goes to the elastic wave propagation in the shell. 
At speed $150 \mathrm{~m} / \mathrm{sec}$, enough of the shell elements around the point of impact fracture that allows full penetrated of the shell by the impactor. The impactor passes through the shell. It delivers some of its kinetic energy to the fractured elements and to the permanent deformation of the surrounding shell elements. Besides the part of energy that goes to the elastic wave propagation in the shell. The impactor passes through the shell structure with the residual kinetic energy.

Figure 7 shows the variation of impactor displacement through the impact velocity and compares the results between our method and LS-Dyna which appear a good agreement.

\section{CONCLUSIONS}

The structural impact model proposed in this paper provides a conceptual framework for better understanding on the finite-element simulation of shell behavior under projectile impact loading. This investigation also explored the suitability of employing load curves to represent the real rate-dependent elasto-plastic nature of shells.

The numerical results comparing our method and LS-DYNA correlate closely in terms of projectile residual velocity and energy absorption. The proposed numerical model is also able to provide an analysis of the shell transverse deflection profile during perforation. Examination of LS-DYNA results after perforation indicates that deformation is more localized under high-velocity impact as predicted by the proposed numerical simulation. It has also been noted that simplifications assumed in determining the variation of dynamic properties in which the strain rate effect is neglected and contact detection is oversimplified is not acceptable if accurate impact solution is needed.

The model provides a fairly accurate representation of the strain-rate-dependent behavior of shells within a limited scope of investigation. It yields a good basis for future work by understanding the methodology adopted and the limitations involved in this study.

\section{REFERENCES}

1. Billon H., "A model for ballistic impact on soft armour." , DSTO Aeronautical \& Maritime Research Laboratory, DSTO-TR-0730, (1998)

2. Hunter SC., "Energy absorbed by elastic waves during impact.", J. Mech. Phys. Solids,No.8,pp 162, (1957)

3. Hutchings IM., "Energy absorbed by elastic waves during plastic impact.", J. Applied Phys, No.12, pp 1819,(1979)

4. Jonas A. Zukas, Theodore Nicholas, Hall F. Swift, "Impact Dynamics", John Wiley \& Sons Inc.,(1982)

5. Karl F. Graff, "Wave Motion in Elastic Solids", Dover Publications, Inc., (1975)

6. Kolsky H., "Stress Waves in Solids", Dover Publications, Inc., (1963)

7. M. ElFarran, "Predication of structural damage due to impact", PhD thesis, Cairo university, (2007)

8. Norman Jones, "Structural Impact", Cambridge University Press,(1989) 
9. Reed J., "Energy losses due to elastic wave propagation during an elastic impact.", J. Applied Phys,No.18, pp 2329, (1985)

10. Tillett J. P. A., "A study of the impact on sphere of plates", Proc. Phys. Soc, No.B69, pp 677, (1954).

Impactor velocity $=10 \mathrm{~m} / \mathrm{s}$ at $\mathrm{t}=202 \mu \mathrm{sec}$ "Elastic deformation"

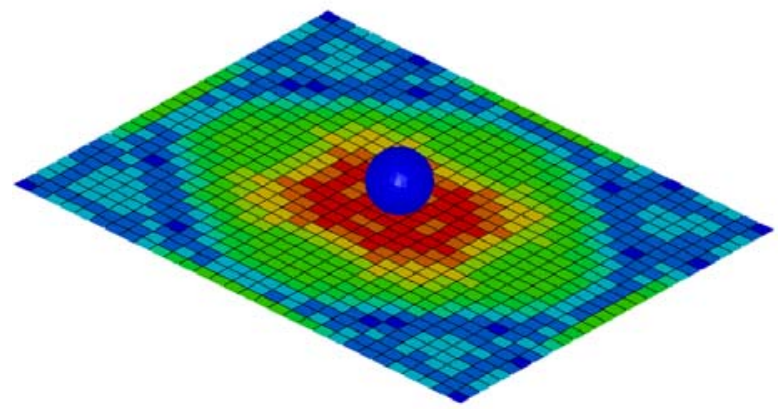

Impactor velocity $=20 \mathrm{~m} / \mathrm{s}$ at $\mathrm{t}=104 \mu \mathrm{sec}$ "Plastic deformation"
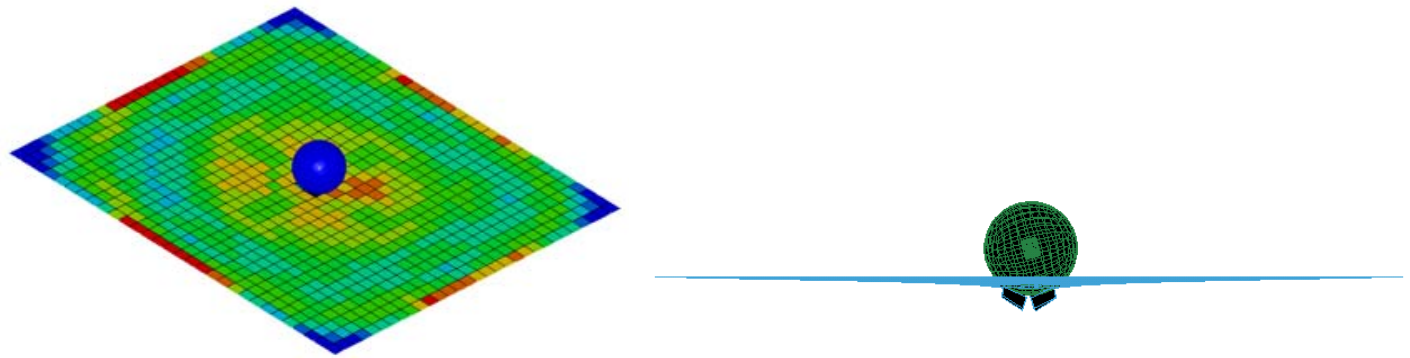

Impactor velocity $=30 \mathrm{~m} / \mathrm{s}$ at $\mathrm{t}=104 \mu \mathrm{sec}$ "Penetrated"
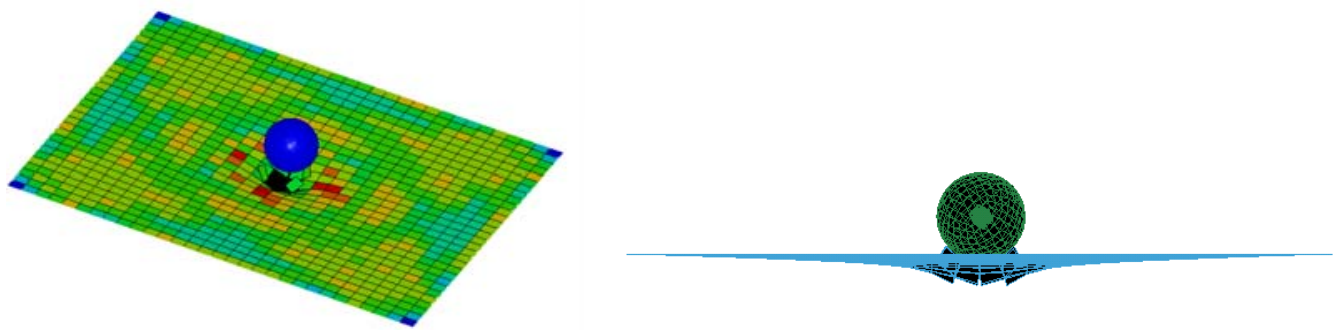

Impactor velocity $=60 \mathrm{~m} / \mathrm{s}$ at $\mathrm{t}=180 \mu \mathrm{sec}$ "Penetrated"
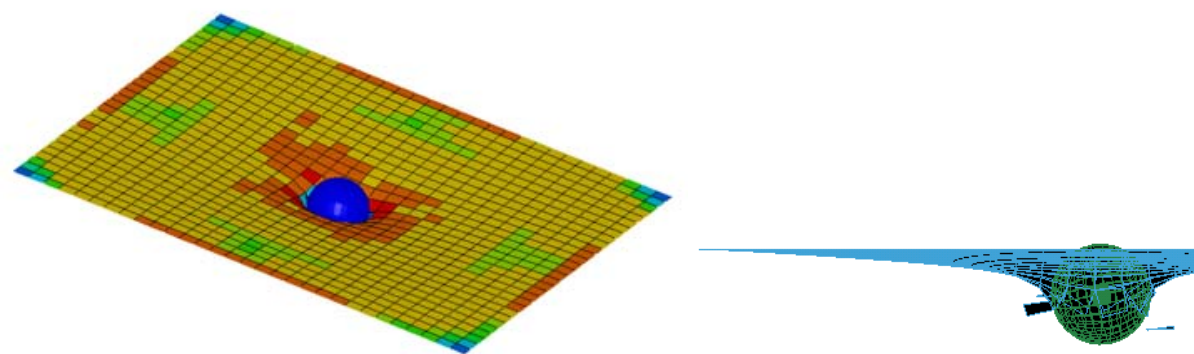

Impactor velocity $=90 \mathrm{~m} / \mathrm{s}$ at $\mathrm{t}=65 \mu \mathrm{sec}$ "Penetrated" 

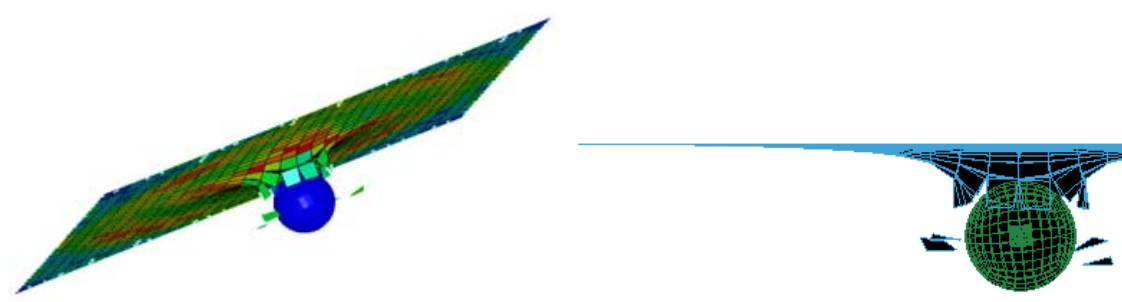

impactor velocity $=150 \mathrm{~m} / \mathrm{s}$ at $\mathrm{t}=35 \mu \mathrm{sec}$ "perforated"

Fig.6. Displacement Profile for different impactor velocities.

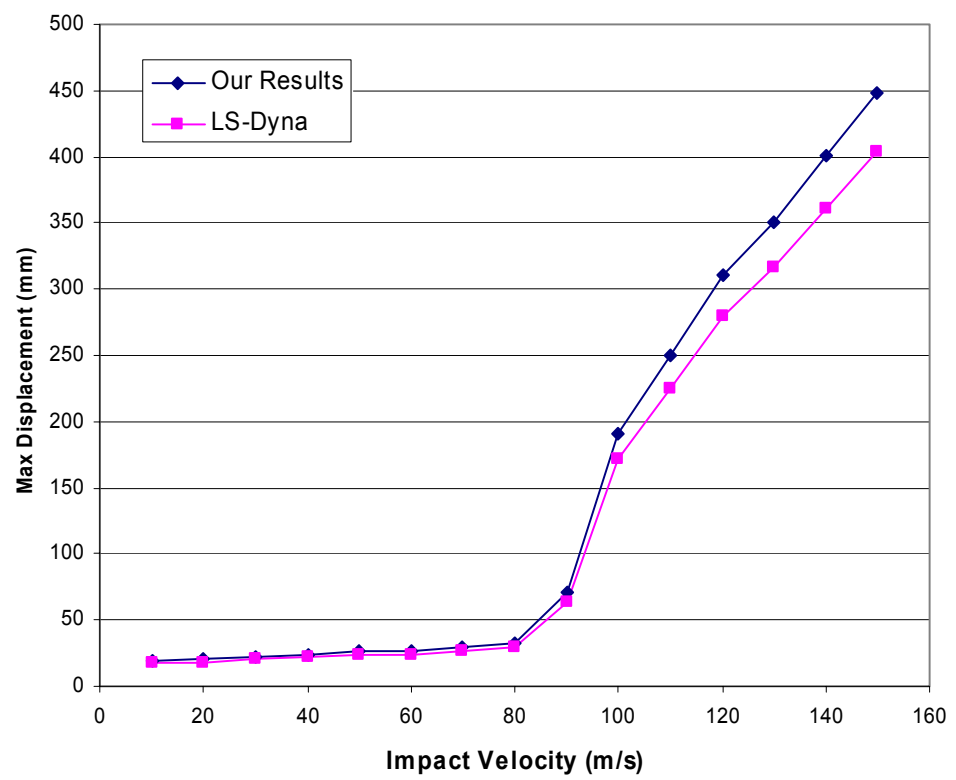

Fig.7. Variation of impactor displacement with impact velocity 